Original Research Paper

\title{
Prevalence and Antibiotic Sensitivity test of Salmonella Serovars from Enteric Fever Suspected Patients Visiting Alka Hospital, Lalitpur
}

\author{
${ }^{1}$ Kalpana Pandey, ${ }^{2}$ Vijay K. Sharma and ${ }^{3}$ Roshani Maharjan \\ ${ }^{I}$ Department of Microbiology, St. Xavier's College, Maitighar, Kathmandu, Nepal \\ ${ }^{2}$ Department of Pathology, Alka Hospital, Lalitpur, Nepal \\ ${ }^{3}$ Department of Microbiology, Tri-chandra Multiple Campus, Kathmandu, Nepal
}

Article history

Received: 22-06-2015

Revised: $20-08-2015$

Accepted: 15-10-2015

Corresponding Author: Kalpana Pandey

Department of Microbiology,

St. Xavier's College,

Maitighar, Kathmandu, Nepal

Email: pandey.kalpana099@gmail.com

\begin{abstract}
Enteric fever is still an important public health problem in developing countries including Nepal. A changing antibiotic susceptibility pattern of Salmonella Typhi and Salmonella Paratyphi A has increased to a great concern. To determine the prevalence and antibiotic sensitivity pattern of $S$. enteric serovars Typhi and Paratyphi from blood specimen in enteric fever suspected culture positive cases. A cross-sectional study was carried out at Alka hospital, lalitpur. Blood culture samples were collected from suspected enteric fever patient and tested microbiologically by standard procedure. AST was based on Kirby disc diffusion method and results were interpreted according to Clinical Laboratory Standards Institute (CLSI) guidelines. Of total $37(6.10 \%)$ Salmonella serotype isolated from 604 blood culture samples, $25(67.57 \%)$ were $S$. Typhi and $12(32.43 \%)$ were $S$. Paratyphi A. Among the culture positive cases, the incidence rate was high in male $70.27 \%$ and the age group of $21-30$ years showed maximum number of growth i.e., $14(37.8 \%)$. Among the tested antibiotics $S$. Typhi was fully susceptible towards Ceftriaxone. In case of $S$. Paratyphi A Ciprofloxacin, Cefriaxone and Cefixime showed 100\% susceptibility. Resistant pattern of Salmonella serovars was seen in higher number 34 $(91.9 \%)$ in the case of Nalidixic acid. Among 37 Salmonella serovars isolated six were found to be Multidrug Resistance (MDR) where four were Salmonella Typhi and two were Salmonella Paratyphi A. Ceftriaxone and Cefixime was the most effective drugs as no isolates were resistant. Ceftriaxone and Cefixime can be the better choice of antibiotic for Salmonella isolates and remains the last drug of choice for $S$. Typhi and $S$. Paratyphi A which are also resistant to the first line agents.
\end{abstract}

Keywords: Enteric Fever, Nalidixic Acid Resistant Salmonella, Multidrug Resistance

\section{Introduction}

Enteric fever is a systemic infection caused by the human adapted pathogens Salmonella enterica serotype Typhi ( $S$. Typhi) and $S$. enterica serotype Paratyphi $(S$. Paratyphi) A, B and C (Crump and Mintz, 2010). Enteric fever remains one of the major public health issues globally, especially in Asia. According to recently revised global estimate, above 22 million cases of typhoid fever occur each year round the world while $90 \%$ of the sufferers are from the South East Asia (Rahman et al., 2011). Its incidence is highest in children and young adults between 5 and 19 years old (WHO, 2007). In context of Nepal, enteric fever caused by Salmonella enterica serovar Typhi and Paratyphi A is the most common clinical diagnosis among febrile patients (Maskey et al., 2008).

A changing antibiotic susceptibility pattern of Salmonella isolates and emergence of Salmonella Paratyphi A as a cause of enteric fever has increased to a great concern. So, this study was undertaken to isolate and identify $S$. Typhi and $S$. Paratyphi and their antibiotic sensitivity pattern which are the major cause of enteric fever in the developing countries like Nepal. 


\section{Methods}

The study was carried out at Alka hospital, Lalitpur from 15th of March to 15th of September, 2014.

Blood culture samples were collected visiting the hospital. Brain-Heart Infusion (BHI) broth was used as enrichment media which supports the growth of all common pathogens causing enteric fever. Collection of blood, incubation and subcultures onto blood agar and Mac-Conkey agar were done as per the standard methods (Cheesbrough, 2006). Suspected non-lactose-fermenting colonies were further processed and identified by biochemical tests like Catalase test, Citrate test, Triple Sugar iron test, Urease test, Motility test, Methyl-Red and Voges-Proskauer test, Gas production test, Sulfide production test and confirmed (Chakraborty, 2007). Antimicrobial susceptibility was determined by the Kirby-Bauer disc diffusion method (Collee et al., 2006). The antibiotic discs used were Cotrimoxazole (25 mcg), Ciprofloxacin (5 mcg), Ceftriaxone (30 $\mathrm{mcg})$, Azithromycin (15 mcg), Nalidixic acid (30 $\mathrm{mcg})$, Amoxycillin (30 mcg), Cefixime $(5 \mathrm{mcg})$ and Ofloxacin $(5 \mathrm{mcg})$. Result was interpreted according to Clinical Laboratory Standards Institute (CLSI) guidelines. Data were entered in Microsoft Excel and analyzed by SPSS version 16.0.

\section{Results}

A total of 604 patients suspected of enteric fever were 37 Salmonella isolates, 25 (67.57\%) were $S$. Typhi and 12 (32.43\%) were $S$. Paratyphi A. Among the culture positive cases, the incidence rate was high in male $70.27 \%$ than female $29.73 \%$ and the age group of 21-30 years showed maximum number of growth i.e., 14. However, the association of organism isolated with respect to different age groups and gender was found to be statistically insignificant $(p>0.05)$. Most of the isolates $(27.0 \%)$ were isolated in the month August and September and the association was statistically insignificant ( $>0.05)$. Ceftriaxone was found to be $100 \%$ effective in all the $S$. Typhi isolates. It was followed by Cefixime (96\%) and Cotrimoxazole with $88 \%$ susceptibility (Table 1-3.2). studied, among them $6.10 \%$ were culture positive. Out of

Table 1. Age wise distribution of Salmonella serovars

\begin{tabular}{lllc}
\hline & Organisms & & \\
Age in years & $S$. Typhi & S. Paratyphi A & Total no \\
\hline $0-10$ & 4 & 4 & 8 \\
$11-20$ & 10 & 3 & 13 \\
$21-30$ & 10 & 4 & 14 \\
$31-40$ & 1 & 0 & 1 \\
$41-50$ & 0 & 1 & 1 \\
\hline
\end{tabular}

Table 1.1. Gender wise distribution of Salmonella serovars

\begin{tabular}{lcll}
\hline & Organism & & \\
& ------------ & \\
Sex of the patient & $S$. Typhi & S. Paratyphi A & Total (\%) \\
\hline Male & 19 & 7 & $26(70.2)$ \\
Female & 6 & 5 & $11(29.7)$ \\
\hline
\end{tabular}

Table 1.2. Month wise distribution of isolates

\begin{tabular}{llll}
\hline & Organism isolated & \\
\cline { 2 - 3 } Months & $S$. Typhi & S. Paratyphi A & Total no (\%) \\
\hline March/April & 3 & 2 & $5(13.5)$ \\
April/May & 3 & 1 & $4(10.8)$ \\
May/June & 4 & 2 & $6(16.21)$ \\
June/July & 4 & 1 & $5(13.56)$ \\
July/August & 5 & 2 & $7(18.91)$ \\
August/Sep. & 6 & 4 & $10(27.0)$ \\
\hline
\end{tabular}

Table 2. Antibiotic susceptibility pattern of Salmonella isolates

\begin{tabular}{|c|c|c|}
\hline \multirow[b]{2}{*}{ Antibiotic used } & \multicolumn{2}{|c|}{ Antibiotic susceptibility Pattern } \\
\hline & $S$. Typhi & S. Paratyphi A \\
\hline Cotrimoxazole & $22(88 \%)$ & $10(83.33 \%)$ \\
\hline Cefixime & $24(96 \%)$ & $12(100)$ \\
\hline Amoxycillin & $19(76 \%)$ & $10(83.33 \%)$ \\
\hline Ciprofloxacin & $15(60 \%)$ & $12(100)$ \\
\hline Azithromycin & $18(72 \%)$ & $8(66.67 \%)$ \\
\hline Nalidixic acid & $1(4 \%)$ & 0 \\
\hline Ceftriaxone & $25(100 \%)$ & $25(100 \%)$ \\
\hline Ofloxacin & $17(68 \%)$ & $9(75 \%)$ \\
\hline
\end{tabular}

Table 3. Multidrug resistant isolates among Salmonella

\begin{tabular}{lllc}
\hline Organism & Number & MDR isolates & Total (\%) \\
\hline S. Typhi & 25 & 4 & 16.0 \\
S. Paratyphi A & 12 & 2 & 16.7 \\
Total & 37 & 6 & 16.2 \\
\hline
\end{tabular}

Table 3.1. Antibiotic susceptibility pattern of multidrug resistant Salmonella Typhi

\begin{tabular}{|c|c|c|c|c|}
\hline \multirow[b]{2}{*}{ Antibiotic used } & \multicolumn{4}{|c|}{ Antibiotic susceptibility pattern of multidrug resistant Salmonella Typhi $(\mathrm{n}=4)$} \\
\hline & $\begin{array}{l}\text { Multidrug resistant } \\
S . \text { Typhi }_{1}\end{array}$ & $\begin{array}{l}\text { Multidrug resistant } \\
S . \text { Typhi }_{2}\end{array}$ & $\begin{array}{l}\text { Multidrug resistant } \\
S . \text { Typhi }_{3}\end{array}$ & $\begin{array}{l}\text { Multidrug resistant } \\
S . \text { Typhi }_{4}\end{array}$ \\
\hline Cotrimoxazole & Resistant & Resistant & Resistant & Sensitive \\
\hline Cefixime & Sensitive & Sensitive & Sensitive & Sensitive \\
\hline Amoxycillin & Resistant & Resiatant & Resistant & Resistant \\
\hline Ciprofloxacin & Sensitive & Resistant & Resistant & Sensitive \\
\hline Azithromycin & Sensitive & Sensitive & Resistant & Resistant \\
\hline Nalidixic acid & Resistant & Resiatant & Resistant & Resistant \\
\hline Ceftriaxone & Sensitive & Sensitive & Sensitive & Sensitive \\
\hline Ofloxacin & Resistant & Resistant & Sensitive & Resistant \\
\hline
\end{tabular}


Table 3.2. Antibiotic susceptibility pattern of multidrug resistant Salmonella Paratyphi A

\begin{tabular}{lll} 
& Antibiotic susceptibility pattern of multidrug resistant Salmonella Paratyphi A & Multidrug resistant Paratyphi A \\
Antibiotic used & - & Sensitive \\
\hline Cotrimoxazole & Multidrug resistant Paratyphi A ${ }_{1}$ & Sensitive \\
Cefixime & Sensitive & Resistant \\
Amoxycillin & Sensitive & Sensitive \\
Ciprofloxacin & Resistant & Resistant \\
Azithromycin & Sensitive & Resistant \\
Nalidixic acid & Sensitive & Sensitive \\
Ceftriaxone & Resistant & Resistant \\
Ofloxacin & Sensitive & Resistant \\
\hline
\end{tabular}

In case of $S$. Paratyphi A all the isolates were sensitive to Ceftriaxone, Ciprofloxacin and Cefixime. Among 37 isolates six isolates were found to be multi drug resistance where 4 were Salmonella Typhi and 2 isolates were Salmonella Paratyphi A.

\section{Discussion}

In this study, out of 604 blood culture samples, 37 $(6.10 \%)$ were culture positive. Similar results have been reported by Acharya et al. (2012; Adhikari et al., 2012) who found 7.6 and $8.99 \%$ culture positive result respectively. However, the low isolation of the pathogen in blood culture may be due to the partial treatment received elsewhere before coming to the hospital Shakva et al. (2008) and our reliance upon a single blood culture for diagnosis.

Out of 37 isolates $25(67.57 \%)$ were $S$. Typhi and 12 (32.43\%) were $S$. Paratyphi A. The most diagnosed enteric fever cases were in the month of August and September. The association was statically insignificant $(p>0.05)$. This may be possibly due to the sewagemediated contamination of water sample during the rainy seasons (WHO, 2003).

Our study showed 100\% susceptibility of Ceftriaxone for both $S$. Typhi and $S$. Paratyphi A strains Raza et al. (2012; Khatiwada, 2006) revealed that Ceftriaxone is found to be one the most effective (100\%) antibiotics against Salmonella Paratyphi A Ceftriaxone remains the last line drug against infections with Ciprofloxacin resistant Salmonella when it is resistant to other first line drug.

Salmonella Typhi and Salmonella Paratyphi A were found to be 72 and $66.67 \%$ susceptible towards Azithromycin respectively. The emerging resistant of Azithromycin has also been reported in India by Choudhary et al. (2013) This may be the result of misuse of Azithromycin that has been propelled due to its oral route of administration, as well as broad-spectrum antimicrobial activity with minimal side effects and interactions (Capoor and Nair, 2010).

The susceptibility pattern of Ofloxacin was $68 \%$ for $S$. Typhi and $75 \%$ for $S$. Paratyphi A respectively. Out of 37 isolates $34(91.9 \%)$ were Nalidixic acid resistant. $S$.
Typhi strains showed higher rate of resistance (92\%) towards Nalidixic acid than S. Paratyphi A (91.67\%). The study shows the emergence of Nalidixic acid resistant strains. It is a serious matter related to the health as most of the hospital at remote areas of Nepal don't rely on the sensitivity test due to unavailability and prescribe Ciprofloxacin and Ofloxacin blindly. Other factors for the emergence of Nalidixic acid include self prescription by patients and incomplete course of treatment Pokharel et al. (2006).

The present study showed that only $2 S$. Paratyphi MDR isolate was found resistant to Amoxycillin, Ofloxacin, Azithromycin and Nalidixic acid. Similarly, 4 MDR isolate was found in $S$. Typhi. Besides being resistant to Amoxycillin, Ofloxacin, Azithromycin and Nalidixic acid, it was found to be resistant to Ciprofloxacin and Cotrimoxazole. In a study carried out in Nepal, all the $S$. Typhi and $S$. Paratyphi A isolates were reported as susceptible until 1998 but during 1999 to 2003 Ciprofloxacin resistance increase $5 \%$ in the $S$. Typhi and $13 \%$ in $S$. Paratyphi A Maskey et al. (2008). This increased resistance reflects the overuse of Ciprofloxacin in the treatment of typhoid, as well as in other unrelated infections. Incomplete treatment may also be a factor contributing to development of resistance.

\section{Conclusion}

Cotrimoxazole and Amoxycillin resistant by Salmonella were found to be minimal, so these drugs may also be useful option for treatment of enteric fever. However, Cefixime and Ceftriaxone was cent percent susceptible towards Salmonella. Hence Cefixime remain as oral drug of choice and Ceftriaxone remain intravenous option for treatment of typhoid fever.

\section{Acknowledgement}

We acknowledge the laboratory team of the department of Microbiology and the members of Pathology department, Alka Hospital for providing us the precious encouragement and generous technical support to complete this research work during the laboratory work. 


\section{Author's Contributions}

Kalpana Pandey: Conducted all laboratory experiments, co-ordinated the data analysis and interpretation of the data and contributed to the manuscript writing.

Vijay K. Sharma: Designed the research conception, organized the study and critically reviewed the manuscript.

Roshani Maharjan: Contributed in the data analysis and interpretation of the data and also assisted in manuscript writing.

\section{Ethics}

The corresponding author confirms that all of the other authors have read and approved the manuscript and no ethical issues involved.

\section{References}

Acharya, D., S. Trakulsomboon, S.K. Madhup and S. Korbsrisate, 2012. Antibiotic susceptibility pattern and the indicator of decreased ciprofloxacin susceptibility of Salmonella enterica serovar Typhi isolated from Dhulikhel Hospital, Nepal. Jpn. J. Infec. Dis., 65: 264-267. PMID: 22627312

Adhikari, D., D. Acharya, P. Shrestha and R. Amatya, 2012. Ciprofloxacin susceptibility of Salmonella enteric serovar Typhi and Paratyphi A from blood samples of suspected enteric fever patients. Int. J. Infect. Microbiol., 1: 9-13. DOI: $10.3126 /$ ijim.v1i1.6938

Capoor, M.R. and D. Nair, 2010. Quinolone and cephalosporin resistance in enteric fever. J. Global Infect. Dis., 2: 258-262.

DOI: $10.4103 / 0974-777 X .68529$

Chakraborty, P., 2007. A Textbook of Microbiology. 1st Edn., New Central Book Agency (P) Ltd, India, ISBN-10: 8173810818 .

Cheesbrough, M., 2006. District Laboratory Practice in Tropical Countries. 2nd Edn., Cambridge University Press, New York, ISBN-10: 0521676312, pp: 440.

Choudhary, A., R. Gopalakrishnan, P.S. Nambi, V. Ramasubramanian and K.A. Ghafur et al., 2013. Antimicrobial susceptibility of Salmonella enterica serovars in a tertiary care hospital in southern India. Indian J. Med. Res., 137: 800-802. PMID: 23703350
Collee, J.G., A.G. Fraber, B.P. Marmion and A. Simmons, 2006. Mackie and McCartney Practical Medical Microbiology. In: Salmonella, Brands, D.A., I.E., Alcamo and D.L. Heymann (Eds.), Infobase Publishing, Philadelphia, ISBN-10: 1438101651, pp: 385-404.

Crump, J.A. and E.D. Mintz, 2010. Global trends in typhoid and paratyphoid fever. Clin. Infect. Dis., 50: 241-246. DOI: 10.1086/649541

Khatiwada, S., 2006. Study of prevalence of enteric fever and assessment of widal test in the diagnosis of typhoid fever. A M.Sc. Thesis, Central Department of Microbiology, Tribhuvan University.

Maskey, A.P., B. Basnyat, G.E. Thwaites, J.I. Campbell and J.J. Farrar et al., 2008. Emerging trends in enteric fever in Nepal: 9124 cases confirmed by blood culture 1993-2003. Trans. Royal Society Tropical Med. Hygiene, 102: 91-95. DOI: $10.1016 /$ j.trstmh.2007.10.003

Pokharel, B.M., J. Koirala, R.K. Dahal, S.K. Mishra and P.K. Khadga et al., 2006. Multidrug-resistant and Extended-Spectrum Beta-Lactamase (ESBL)producing Salmonella enterica (serotypes Typhi and Paratyphi A) from blood isolates in Nepal: Surveillance of resistance and a search for newer alternatives. Int. J. Infect. Dis., 10: 434-438. DOI: $10.1016 /$ j.ijid.2006.07.001

Rahman, A.K.M.M., M. Ahmad, R.S. Begum, M.Z. Hossain and S.A. Hoque et al., 2011. Prevalence of typhoid fever among the children in a semi urban area of Bangladesh. J. Dhaka Med. College, 20: 37-43. DOI: 10.3329/jdmc.v20i1.8570

Raza, S., R. Tamrakar, C.P. Bhatt and S.K. Joshi, 2012. Antimicrobial susceptibility patterns of Salmonella Typhi and salmonella paratyphi A in a tertiary care hospital. J. Nepal Health Res. Counc., 22: 214-217. PMID: 23281454

Shakva, K.N., M.R. Baral and R. Shrestha, 2008. A study of atypical manifestations of enteric fever in children. J. Nepal Health Res. Counc., 6: 1-4.

WHO, 2003. Background document: The diagnosis, treatment and prevention of typhoid fever. World Health Organization, Geneva.

WHO, 2007. Typhoid fever. World Health Organization. 